\title{
Sodium abundances in nearby disk stars ${ }^{\star \star \star}$
}

\author{
J. R. Shi ${ }^{1,2}$, T. Gehren ${ }^{2}$, and G. Zhao ${ }^{1,2}$ \\ 1 National Astronomical Observatories, Chinese Academy of Sciences, Beijing 100012, PR China \\ e-mail: sjr@bao.ac.cn \\ ${ }^{2}$ Institut für Astronomie und Astrophysik der Universität München, Scheinerstrasse 1, 81679 München, Germany
}

Received 5 February 2004 / Accepted 4 May 2004

\begin{abstract}
We present sodium abundances for a sample of nearby stars. All results have been derived from NLTE statistical equilibrium calculations. The influence of collisional interactions with electrons and hydrogen atoms is evaluated by comparison of the solar spectrum with very precise fits to the Na I line cores. The NLTE effects are more pronounced in metal-poor stars since the statistical equilibrium is dominated by collisions of which at least the electronic component is substantially reduced. The resulting influence on the determination of sodium abundances is in a direction opposite to that found previously for $\mathrm{Mg}$ and Al. The NLTE corrections are about -0.1 in thick-disk stars with $[\mathrm{Fe} / \mathrm{H}] \sim-0.6$. Our $[\mathrm{Na} / \mathrm{Fe}]$ abundance ratios are about solar for thick- and thin-disk stars. The increase in $[\mathrm{Na} / \mathrm{Fe}]$ as a function of $[\mathrm{Fe} / \mathrm{H}]$ for metal-rich stars found by Edvardsson et al. (1993) is confirmed. Our results suggest that sodium yields increase with the metallicity, and quite large amounts of sodium may be produced by AGB stars. We find that $[\mathrm{Na} / \mathrm{Fe}]$ ratios, together with either $[\mathrm{Mg} / \mathrm{Fe}]$ ratio, kinematic data or stellar evolutionary ages, make possible the individual discrimination between thin- and thick-disk membership.
\end{abstract}

Key words. line: formation - line: profiles - stars: abundances - stars: late-type - Galaxy: evolution

\section{Introduction}

The ${ }^{23} \mathrm{Na}$ nucleus contains an extra neutron that makes its synthesis deviate from that of the $\alpha$-nuclei such as ${ }^{24} \mathrm{Mg}$. So the abundance ratios $[\mathrm{Na} / \mathrm{Fe}]$ and $/$ or $[\mathrm{Na} / \mathrm{Mg}]$ in stars are a potential source of information concerning the history of Galactic nucleosynthesis.

It is expected that sodium is made during carbon and neon burning in massive stars, and it would therefore be ejected by Type II supernova (SN II). Type Ia supernova, on the other hand, produce little sodium (Tsujimoto et al. 1995). However, ${ }^{23} \mathrm{Na}$ can be synthesized as a primary or secondary element. In the first case ${ }^{23} \mathrm{Na}$ would be produced directly in the carbon burning process, and the production rate would be independent of the metal content of the proceeding stellar generation. In the second process, where ${ }^{23} \mathrm{Na}$ is produced by an excess of neutrons, the production rate would depend on that neutron excess which in turn would be determined by the pre-existing metal abundance. In the latter case ${ }^{23} \mathrm{Na}$ would be expected to display an underabundance compared with $\mathrm{Fe}$ or $\mathrm{Mg}$ in the most metal-poor stars. Although significant progress has been made in nucleosynthesis calculations, theoretical SN yields of

\footnotetext{
* Based on observations collected at the Germany-Spanish Astronomical Center, Calar Alto, Spain.

$\star \star$ Tables 2 and 3 are only available in electronic form at http://www.edpsciences.org
}

sodium have not yet converged to consistency among models from different authors (Woosley \& Weaver 1995; Limongi et al. 1998; Umeda et al. 2000). Also some synthesis of sodium in intermediate-mass stars can occur in the hydrogenburning shell through the neon-sodium cycle (Denissenkov \& Denissenkova 1990; Mowlavi 1999).

The observational picture for this element is complicated. The early studies report that sodium scales with iron at all metallicities (e.g. the review of Timmes et al. 1995; Goswami \& Prantzos 2000). However, new studies do not support this classical picture. For metal-poor stars, Pilachowski et al. (1996) analyzed a sample of 60 halo subgiants, giants and horizontal branch stars in the interval $-3 \leq[\mathrm{Fe} / \mathrm{H}] \leq-1$, and found a small $[\mathrm{Na} / \mathrm{Fe}]$ deficiency of -0.17 dex in the mean and that bright field halo giants do not show the excess of sodium found in their globular cluster counterparts. Baumüller et al. (1998) performed a NLTE analysis of sodium and reported a clear trend of decreasing $[\mathrm{Na} / \mathrm{Fe}]$ with decreasing metallicity. Similarly, Stephens (1999) found that $[\mathrm{Na} / \mathrm{Fe}]$ decreases as one goes from $[\mathrm{Fe} / \mathrm{H}]=-1$ to $[\mathrm{Fe} / \mathrm{H}]=-2$, and thus shows the theoretically expected behaviour of odd- $Z$ elements. Carretta et al. (2000) and Gratton et al. (2003) also found that sodium is overdeficient in stars with $[\mathrm{Fe} / \mathrm{H}] \leq-1$; this result was confirmed by very recent work of Gehren et al. (2004).

For disk stars, Edvardsson et al. (1993) used highresolution, low-noise spectra to determine sodium abundance 
in $189 \mathrm{~F}$ and $\mathrm{G}$ stars. Their LTE analysis suggested that the $[\mathrm{Na} / \mathrm{Fe}]$ ratio is solar and varies very little with metallicity, which was confirmed by Chen et al. (2000). Based on 5682/5688 and 6154/6160 lines, Prochaska et al. (2000) measured sodium abundance in their thick-disk stars and found that all the stars show mildly enhanced $[\mathrm{Na} / \mathrm{Fe}]$ and there is a mild trend with metallicity (see also Gehren et al. 2004).

Concerning the most metal-rich stars in the galactic disk, the study by Edvardsson et al. (1993) raised a number of new questions. They found sodium relative to iron versus $[\mathrm{Fe} / \mathrm{H}]$ shows an increase for $[\mathrm{Fe} / \mathrm{H}]>0.0$ (see also Feltzing \& Gustafsson 1998, their Fig. 8). Is the "upturn" real? Large starto-star scatter was encountered. Could this scatter to be reduced by using better abundance criteria?

It is known that $[\mathrm{Fe} / \mathrm{H}]$ distributions of the halo, thick- and thin-disk stars reveal considerable overlap, so it can not allow unequivocal classification for many stars without reference to other criteria such as age or kinematics. Fuhrmann (1998, 2002) suggested that the thick-disk stars represent the principal population for the early Galactic evolution, while the stellar halo contributes by only minute amounts (see also Ryan \& Smith 2003). Considering the general kinematics, there may be a significant overlap of all populations (Gehren et al. 2004). Gratton et al. (1996, 2000, 2003) have shown that thick-disk stars are more $[\mathrm{Mg} / \mathrm{Fe}]$ enhanced than the thin-disk stars at the same $[\mathrm{Fe} / \mathrm{H}]$ value. This trend has also been reported by Fuhrmann (1998; see also Prochaska et al. 2000; Reddy et al. 2003). Finally, Mashonkina et al. (2003) have given evidence that $[\mathrm{Eu} / \mathrm{Mg}]$ ratio allows the individual discrimination of halo stars from thick-disk stars, and similar behaviour is found for the $[\mathrm{Al} / \mathrm{Mg}]$ ratio by Gehren et al. (2004).

The present work is based on a sample of nearby stars and aims at exploring their $[\mathrm{Na} / \mathrm{Fe}]$ abundance ratios applying full spectrum synthesis based on level populations calculated from the statistical equilibrium equations. In Sect. 2 we present the observational techniques, while the atmospheric models and stellar parameters are discussed in Sect. 3. NLTE line formation is discussed in Sect. 4. The discussion is presented in Sect. 5, and the conclusions are found in the last section.

\section{Observations}

Our approach towards a representative abundance investigation is aimed at analyzing a volume-complete sample of the $\mathrm{F}, \mathrm{G}$ and early $\mathrm{K}$ dwarfs in the disk population, north of declination $\delta=-15^{\circ}$ and within $25 \mathrm{pc}$ of the Sun (see Fuhrmann 2004, for details). The spectra of our samples were obtained through the years 1995 to 2000 by Klaus Fuhrmann with the fiber-coupled Cassegrain échelle spectrograph FOCES (Pfeiffer et al. 1998) mounted at the $2.2 \mathrm{~m}$ telescope of the Calar Alto Observatory. Only few of them were exposed to a $1024^{2} 24 \mu \mathrm{CCD}$ with $\lambda / \Delta \lambda \sim 40000$, and their wavelength ranges are limited to $4000-7000 \AA$, whereas the standard configuration was a $2048^{2} 15 \mu \mathrm{CCD}$ that covered 4000-9000 with $\lambda / \Delta \lambda \sim 60000$. All stars were observed at least twice with a signal-to-noise ratio of at least $S / N \sim 150$ up to $S / N \sim 400$ (see Fuhrmann 1998, 2000, for details).

\section{Atmospheric models and stellar parameters}

\subsection{Model atmospheres}

Our analyses are all based on the same type of atmospheric model, irrespective of temperature, gravity or metal abundance. We use line-blanketed LTE model atmospheres, generated as discussed by Fuhrmann et al. (1997). The main characteristics are:

- the iron opacity was calculated with the improved meteoritic value $\log \varepsilon_{\mathrm{Fe}}=7.51$;

- opacities for metal-poor stars with $[\mathrm{Fe} / \mathrm{H}]<-0.6$ were calculated using $\alpha$-element $(\mathrm{O}, \mathrm{Ne}, \mathrm{Mg}, \mathrm{Si}, \mathrm{S}, \mathrm{Ar}, \mathrm{Ca}$ and $\mathrm{Ti}$ ) abundances enhanced by $0.4 \mathrm{dex}$;

- the mixing-length parameter $l / H_{p}$ was adopted to be 0.5 (see Fuhrmann et al. 1993).

\subsection{Stellar parameters}

For most of the stars we adopt the stellar parameters determined by Fuhrmann $(1998,2000)$, where the effective temperatures are derived from the wings of the Balmer lines; surface gravities are taken from the strong line wings of the $\mathrm{MgIb}$ triplet and compared with values obtained from Hipparcos parallaxes. Iron abundances are based on Fe II lines, and the microturbulence velocities are estimated by requesting that the iron abundance derived from Fe II lines should not depend on equivalent width. The uncertainties for the temperature, surface gravity, metal abundance and microturbulence velocities are generally assumed to be $\pm 80 \mathrm{~K}, 0.1 \mathrm{dex}, 0.2 \mathrm{~km} \mathrm{~s}^{-1}$ and 0.07 dex respectively (see Fuhrmann 1998, 2000, for details). For Procyon the parameters were taken from Korn et al. (2003), where the temperatures are again from the wings of Balmer lines; surface gravities are based on the HIPPARCOS parallaxes, and iron abundances are taken from NLTE calculations.

Following Fuhrmann's work, we employ the macroturbulence parameters $\zeta$ in the radial-tangential form and adopt values as described in Gray (1984). The projected rotational velocity is obtained as the residual to the observed line profiles, after having removed the known instrumental profile obtained from the Moon spectra.

\section{NLTE line formation}

\subsection{Atomic model}

The model of $\mathrm{Na}$ we adopt goes back to the atomic properties documented in Baumüller et al. (1998), where most of the problems have been described in detail. In this paper, we extend the number of levels to $n=15$. The model is closed with the $\mathrm{Na}^{+}$ground state. Fine structure splitting is neglected with the exception of $3 \mathrm{p}^{2} \mathrm{P}_{1 / 2}^{0}$ and $3 \mathrm{p}^{2} \mathrm{P}_{3 / 2}^{0}$. Part of that model was shown by Baumüller et al. (1998, see their Fig. 2). Energy levels are taken from Martin \& Zalubas (1981) if available. For levels with $l \geq 4$ hydrogenic approximations are used. All bound-free radiative cross-sections have been included from close-coupling calculations of Butler (1993) and Butler et al. (1993). 
Table 1. Atomic data of Na lines. $\log g f$ values and damping constants have been determined from solar spectrum fits.

\begin{tabular}{rcrr}
\hline \hline$\lambda[\AA]$ & Transition & $\log g f$ & $\log C_{6}$ \\
\hline 5682.642 & $3 \mathrm{p}^{2} \mathrm{P}_{1 / 2}^{0}-4 \mathrm{~d}^{2} \mathrm{D}_{3 / 2}$ & -0.72 & -30.05 \\
5688.214 & $3 \mathrm{p}^{2} \mathrm{P}_{3 / 2}^{0}-4 \mathrm{~d}^{2} \mathrm{D}_{5 / 2}$ & -0.47 & -30.05 \\
5889.959 & $3 \mathrm{~s}^{2} \mathrm{~S}_{1 / 2}-3 \mathrm{p}^{2} \mathrm{P}_{1 / 2}^{0}$ & +0.11 & -31.60 \\
5895.932 & $3 \mathrm{~s}^{2} \mathrm{~S}_{1 / 2}-3 \mathrm{p}^{2} \mathrm{P}_{3 / 2}^{0}$ & -0.19 & -31.60 \\
6154.228 & $3 \mathrm{p}^{2} \mathrm{P}_{1 / 2}^{0}-5 \mathrm{~s}^{2} \mathrm{~S}_{1 / 2}$ & -1.57 & -29.78 \\
6160.751 & $3 \mathrm{p}^{2} \mathrm{P}_{3 / 2}^{0}-5 \mathrm{~s}^{2} \mathrm{~S}_{1 / 2}$ & -1.28 & -29.78 \\
8183.260 & $3 \mathrm{p}^{2} \mathrm{P}_{1 / 2}^{0}-3 \mathrm{~d}^{2} \mathrm{D}_{3 / 2}$ & 0.28 & -30.73 \\
8194.800 & $3 \mathrm{p}^{2} \mathrm{P}_{3 / 2}^{0}-3 \mathrm{~d}^{2} \mathrm{D}_{5 / 2}$ & 0.49 & -30.73 \\
\hline
\end{tabular}

To establish the influence of deviations from LTE on the ionization equilibrium of sodium, we have chosen the reference stars of Korn et al. (2003). The full analysis of the solar spectrum (Kurucz et al. 1984) and those of the reference stars allows a reasonable choice of the hydrogen collision enhancement factor resulting in $S_{\mathrm{H}}=0.05$ for $\mathrm{Na}$, found by iteration together with all basic stellar paraeters. This number is the same as in the previous work of Baumüller et al. (1998).

All calculations have been carried out with a revised version of the DETAIL program (Butler \& Giddings 1985) using accelerated lambda iteration (see Gehren et al. 2001, 2004, for details).

\subsection{Atomic line data}

As our primary choice we take the oscillator strengths from the NIST data base. We note that the $g f$ values are similar to those adopted by Takada et al. (2003), the average difference $\log g f$ (this work) $-\log g f$ (Takeda) is $-0.02 \pm 0.04$. For the line pair of 6154/6160, our $g f$ values are same as the values adopted by Chen et al. (2000), but slightly higher than the values of Edvardsson et al. (1993) and Feltzing \& Gustafsson (1998), the difference is about 0.04 . However, in our present analysis the absolute value of the oscillator strengths is unimportant because the abundances are evaluated in a fully differential way with respect to the sun.

Collisional broadening through van der Waals interaction with hydrogen atoms is important for strong $\mathrm{Na} \mathrm{D}$ lines. As already pointed out by Gehren et al. $(2001,2004)$ the resulting values of the van der Waals damping constants are mostly near those calculated according to Anstee \& O'Mara's $(1991,1995)$ tables. Fitting the solar profiles with a common Na abundance then shows that our damping constants for the resonance lines are roughly a factor of 2 below those calculated. The adopted line data ( $g f$ values and van der Waals damping constants) are given in Table 1.

\subsection{Sodium abundances}

The abundance determinations for our program stars are made using spectral synthesis. The synthetic spectra are convolved with macroturbulence, rotational and instrumental broadening profiles, in order to match the observed spectral lines.

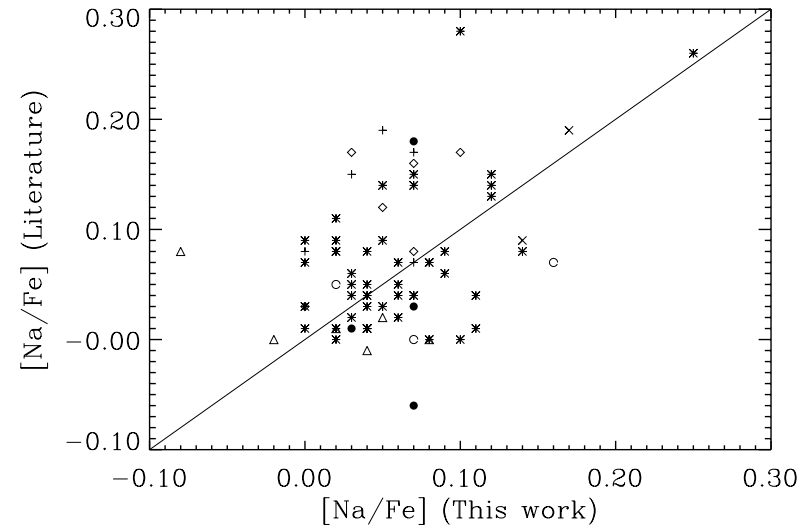

Fig. 1. Comparison of derived $[\mathrm{Na} / \mathrm{Fe}]$ for stars in common with other studies. Filled circles $(\bullet)$ are from Baumüller et al. (1998); crosses $(\times)$ are from Feltzing \& Gustafsson (1998); asterisks $(*)$ are from Carretta et al. (2000); pluses (+) are from Fulbright (2000); diamonds ( $\diamond)$ are from Gratton et al. (2003); open circles (o) are from Takeda et al. (2003); triangles $(\triangle)$ are from Reddy et al. (2003).

Based on 5682/5688, 5890/5896 and 6154/6160 line pairs, our abundance results are obtained from profile fits. However, for $\mathrm{Na} D$ lines, as pointed out by Baumüller et al. (1998), even in the solar atmosphere the inner cores of the lines are affected by deviations from LTE. We note that even for some metal-poor stars, only the line wings can be fitted in LTE. So the sodium abundances are obtained until a best fit to the observed line wings is provided for the LTE analyses. Considering the NLTE abundances, out results do not show large abundance differences between different lines (however, see Takeda et al. 2003). The final abundance scatter of single line is between 0.01 and 0.06 with $\langle\sigma[\mathrm{Na} / \mathrm{Fe}]\rangle=0.020 \pm 0.007$ for all stars. The derived abundances are presented in Table 2. Complete results for individual lines of all stars can be obtained from the author on request.

\subsection{Comparison with other works}

Sodium abundances have been determined by several groups including both LTE and NLTE analyses. In Fig. 1 we compare the $[\mathrm{Na} / \mathrm{Fe}]$ values (NLTE) determined in this paper with those from the literature. The agreement is generally good. However, a closer inspection reveals some systematic differences. In the remaining part of this section we will briefly discuss some possible reasons for these differences.

Baumüller et al. (1998)

Baumüller et al. (1998) performed a full NLTE line formation for a sample of metal-poor stars. We have four stars in common with theirs. Our NLTE results are very much in agreement with theirs, as shown in Fig. 1. The average differences between our $[\mathrm{Na} / \mathrm{Fe}]$ and theirs are $0.02 \pm 0.10$. We note that the considerable scatter is mostly due to the different stellar parameters adopted.

\section{Feltzing \& Gustafsson (1998)}

In this study of $47 \mathrm{G}$ and $\mathrm{K}$ metal-rich stars, they confirmed the increase of $[\mathrm{Na} / \mathrm{Fe}]$ as a function of $[\mathrm{Fe} / \mathrm{H}]$ found previously 
by Edvardsson et al. (1993). Their results were determined from 6154/6160 lines, the $g f$ values adopted in this study are slightly lower than ours, the differences are $-0.02 /-0.05$, respectively. The sodium abundance determined by this study is in good agreement with ours. For three stars in common, the average difference is $0.01 \pm 0.04$.

Carretta et al. (2000)

This work reanalysed the data of Edvardsson et al. (1993) including a NLTE correction. They found no large differences from the LTE results of Edvardsson et al., which is shown in their Fig. 9. The $g f$ values of the 6154/6160 lines adopted by Edvardsson et al. (1993) are slightly lower than ours, the differences are -0.04 and -0.03 , respectively. The results of Carretta et al. (2000) are very much in agreement with ours. For the 46 common stars from Edvardsson et al. (1993), the average difference between ours and theirs is $-0.01 \pm 0.05$.

\section{Fulbright (2000, 2002)}

This analysis deals with a large number of metal-poor stars, of which five stars are in common with our sample. As pointed out by Gehren et al. (2004), there are large differences in stellar parameters; on average, the typical difference in temperatures for the individual star is about $150 \mathrm{~K}$, and there are a few stars for which differences may be much larger. Fulbright's abundances are slightly larger than ours (see Fig. 1). For the five stars in common with our list we obtain $\overline{\Delta[\mathrm{Na} / \mathrm{Fe}]}=$ $-0.09 \pm 0.05$. We find that the largest difference $(\sim 0.4 \mathrm{dex})$ comes from warm metal-poor stars such as HD 284248. We note that considerable discrepancies remain even after correcting for the difference in effective temperatures. As these stars are metal-poor, they may suffer from relatively large NLTE effects (see Sect. 5.1).

\section{Gratton et al. (2003)}

The authors considered NLTE corrections and reported the sodium abundances for 150 field subdwarfs and subgiants. Our results are mostly in agreement with theirs. For the six stars in common, the average difference is $0.07 \pm 0.05$.

Takeda et al. (2003)

Very recently, Takeda et al. (2003) performed a NLTE analysis of sodium abundances for disk and halo stars. They reanalysed the data from Chen et al. (2000). Their results vary from line to line (see their Tables 4 and 5). The results for the 6154/6160 lines show no large differences from those of Chen et al. (see their Fig. 5c). Our $[\mathrm{Na} / \mathrm{Fe}]$ values are in agreement with theirs for the lines of $6154 / 6160 \AA$ for the three stars in common; the average difference is $0.04 \pm 0.06$. As already pointed out by Takeda et al., this line pair is not very sensitive to the NLTE effects.

\section{Reddy et al. (2003)}

This sample consists of $187 \mathrm{~F}$ and $\mathrm{G}$ dwarfs. The sodium abundances were based on 6154/6160 lines, the $g f$ values adopted in their work are nearly same as those we adopted. A very close star by star agreement between the $[\mathrm{Na} / \mathrm{Fe}]$ ratios derived here and those measured by Reddy et al., no difference exceeds 0.20 dex. For the six stars in common, we obtain
$\overline{\Delta[\mathrm{Na} / \mathrm{Fe}]}=0.00 \pm 0.08$. The large scatter is due to HD 218470 ( $0.16 \mathrm{dex})$. This is a high $v \sin i\left(13.1 \mathrm{~km} \mathrm{~s}^{-1}\right)$, high temperature $\left(T_{\text {eff }}=6407\right)$ and low gravity $(\log g=4.02)$ star. For this star their temperature is about $70 \mathrm{~K}$ higher than ours.

\section{Discussion}

\subsection{NLTE effects}

The abundance analyses of sodium clearly show the NLTE effect. There is a tendency that the NLTE effect is large for warm metal-poor subgiant stars, as would be expected. Baumüller et al. (1998) show in their Fig. 11 that the NLTE line cores for the Na D lines are much deeper than in LTE in metaldeficient stars. In LTE abundance analyses this is compensated by simply increasing the sodium abundance until the observed equivalent width is reproduced. Such results are displayed in Table 3 of Baumüller et al. (see also their Table 2). The results confirm, starting from the solar abundance towards the lower metallicity, that the NLTE abundance effect resulting from the $\mathrm{Na} \mathrm{D}$ lines increases reaching a maximum near $[\mathrm{Fe} / \mathrm{H}]=-2$. LTE abundances can be significantly different from their NLTE counterparts, with differences reaching more than 0.6 dex in extreme cases. In Table 3 it is evident that the NLTE effects are systematically stronger for the hotter models, which is in agreement with the statistical equilibrium of both aluminium and magnesium (Baumüller et al. 1997; Zhao \& Gehren 2000). As expected, the strongest departures from LTE are found for models with high temperature and low metallicity. The reduction of surface gravity results in a decreased efficiency of collisions with electrons and hydrogen atoms, which again leads to stronger NLTE effects. In Fig. 2, the differences between LTE and NLTE analyses for our program stars are plotted as a function of metal abundance, temperature and luminosity.

It should be noted that the NLTE effects differ from line to line, reflecting their individual properties. For example, the $8183 / 8194$ lines show relatively large NLTE effects compared to the other lines at similar line-strength. From Table 3, we can see the importance of the NLTE effect on different lines in sodium abundance determination:

- the weak 6154/6160 lines show the smallest NLTE abundance effects $(\leq 0.1$ dex $)$ for the disk stars, so they are the best abundance indicators for LTE analyses of moderately metal-poor stars.

- the NLTE corrections for 5682/5688 lines are relatively small ( $\sim 0.1$ dex) for dwarfs; but they increase for subgiants $(>0.1 \mathrm{dex})$. Therefore these lines should no longer be analyzed with LTE;

- for the 5890/5896 lines, which are important for determining the sodium abundances of very metal-deficient stars, it is necessary to take account of the NLTE effects except for very cool and metal-rich dwarfs. Especially for warm metal-poor giants, the NLTE correction can reach 0.6 dex (see Table 3 for details);

- the 8183/8194 lines are also important for determining the sodium abundances of very metal-deficient stars because of their strength. We suggest that, even for cool metal-rich stars, the NLTE effects should be taken into account. 

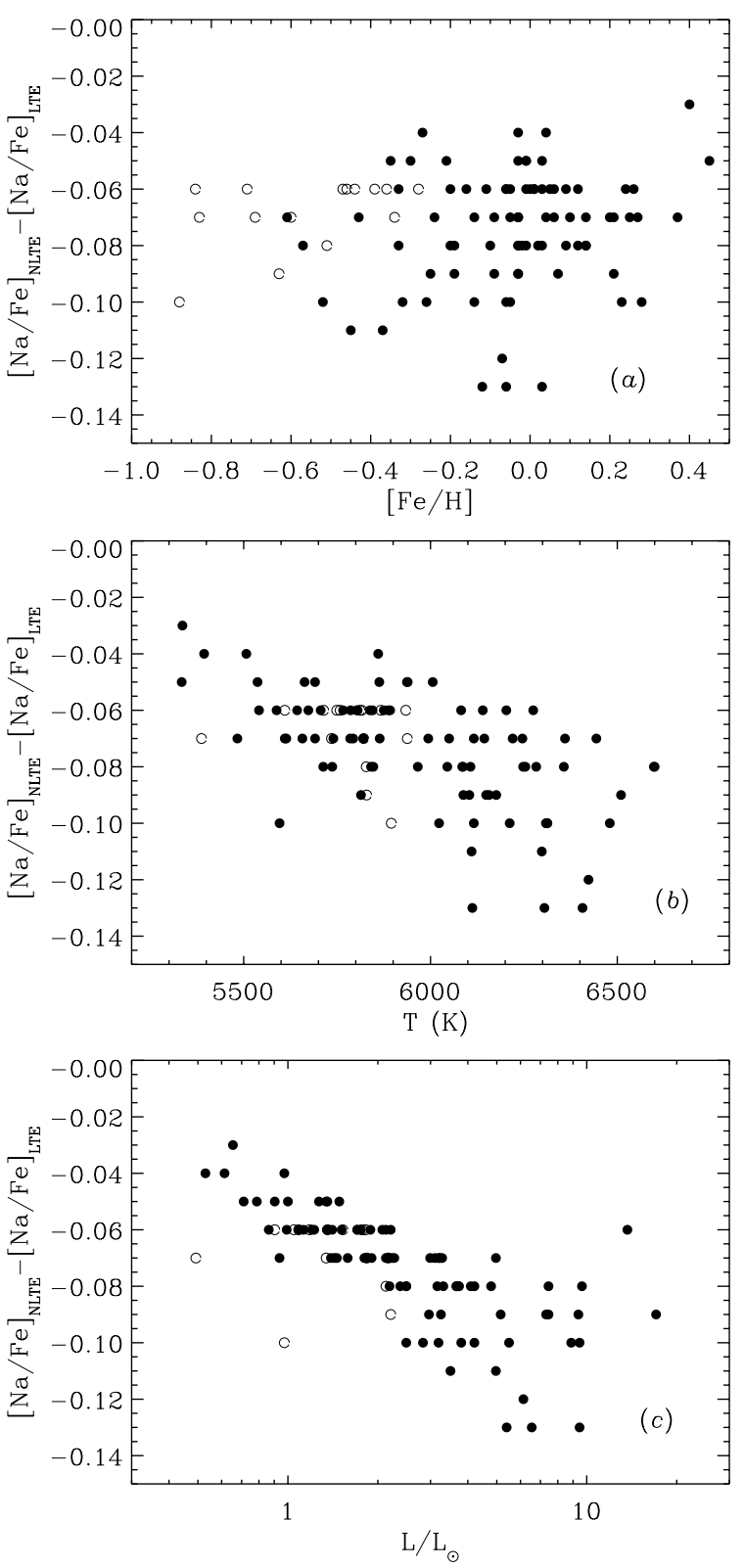

Fig. 2. Difference of $[\mathrm{Na} / \mathrm{Fe}]$ abundance ratios calculated under NLTE and LTE assumptions as a function of metal abundance $\mathbf{a}$ ), temperature b) and luminosity c). Filled circles refer to the thin-disk stars, open circles refer thick-disk stars.

We confirm that departures from LTE of the Na level populations appear to be larger for warm and giant stars, which was also found by Baumüller et al. (1998) and Takeda et al. (2003).

A warning is appropriate that Table 3 should not be used for LTE abundance corrections if precise $\mathrm{Na}$ abundances are aimed at. The data may vary considerably whenever the microturbulence velocity deviates from $1 \mathrm{~km} \mathrm{~s}^{-1}$, and any simple correction may result in an increased scatter. The reference to $[\mathrm{Na} / \mathrm{Fe}]=0$ may also be a bad approximation, particularly in metal-poor stars.

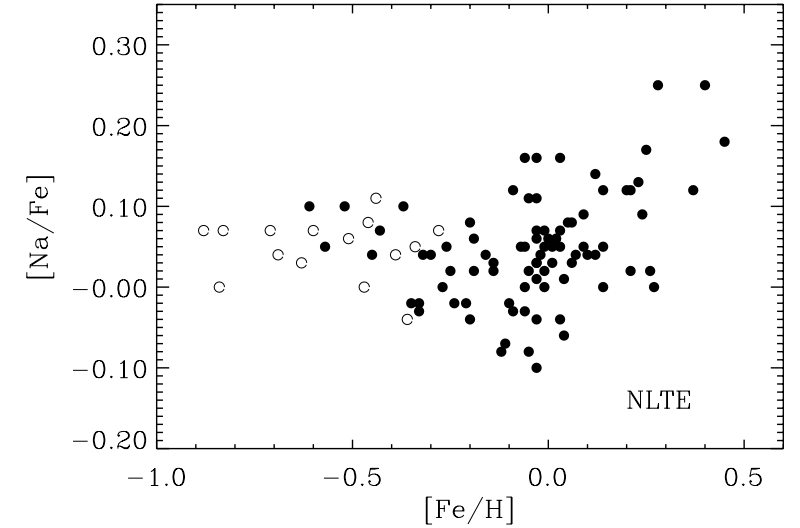

Fig. 3. Abundance ratios $[\mathrm{Na} / \mathrm{Fe}]$ for NLTE analyses. The meaning of the symbols is the same as in Fig. 2.

\subsection{Sodium abundance and nucleosynthesis in the Galaxy}

The variation of $[\mathrm{Na} / \mathrm{Fe}]$ with the stellar metal abundance $[\mathrm{Fe} / \mathrm{H}]$ contains information about the chemical evolution of the Galaxy. Figure 3 displays the run of $[\mathrm{Na} / \mathrm{Fe}]$ ratio (calculated in NLTE) with the overall metal abundance for all stars considered in this paper. One important feature that can be seen from Fig. 3 is that the bulk of the sodium abundances scale approximately as iron in thin-disk stars, with a small overabundance for the thick-disk stars. The "upturn" in $[\mathrm{Na} / \mathrm{Fe}]$ versus $[\mathrm{Fe} / \mathrm{H}]$ for metal-rich disk stars, as observed by Edvardsson et al. (1993), is also reproduced ${ }^{1}$.

Using magnesium instead of iron as the reference can remove Type Ia $\mathrm{SNe}$ from consideration, because nearly all the sodium and magnesium are produced in massive stars (Timmes et al. 1995). Calculations of nucleosynthsis for SNe Ia show that they should produce only very little amounts of sodium (Nomoto et al. 1997). The odd-even effects will be enhanced using magnesium as the basis of comparison. The overall behaviour of $[\mathrm{Na} / \mathrm{Mg}]$ ratios versus $[\mathrm{Mg} / \mathrm{H}]$ is therefore shown in Fig. 4 where the $\mathrm{Mg}$ abundances are taken from Fuhrmann (1998, 2000). We confirm Arnett's (1971) prediction that $[\mathrm{Na} / \mathrm{Mg}]$ ratios decline with decreasing metallicity. This suggests that sodium abundances may be more closely coupled to chemical evolution history, with a gradual enrichment of $[\mathrm{Na} / \mathrm{Mg}]$ from thick to thin disk.

Presently, the source of sodium remains to be confirmed. Thus it is difficult to use sodium as a probe of Galactic chemical evolution until the effects of individual stars can be quantified. Theoretical sodium yields of SN II have not yet converged to consistency among models of different authors (Woosley \& Weaver 1995; Umeda et al. 2000). Umeda et al. (2000) show the yields of sodium are much less for $Z=0$ compared to the $Z=0.02$ for a $20 M_{\odot}$ model, while Woosley \& Weaver (1995) predict that for a $22 M_{\odot}$ SN model the amount of

1 We have 47 stars common with the work of Edvardsson et al. (1993). We note that more metal-rich stars are included in our sample. The upturn in $[\mathrm{Na} / \mathrm{Fe}]$ vs. $[\mathrm{Fe} / \mathrm{H}]$, as observed by Edvardsson et al. is reproduced and reinforced. We confirm that the upturn is real (also see Feltzing \& Gustafsson 1998). 


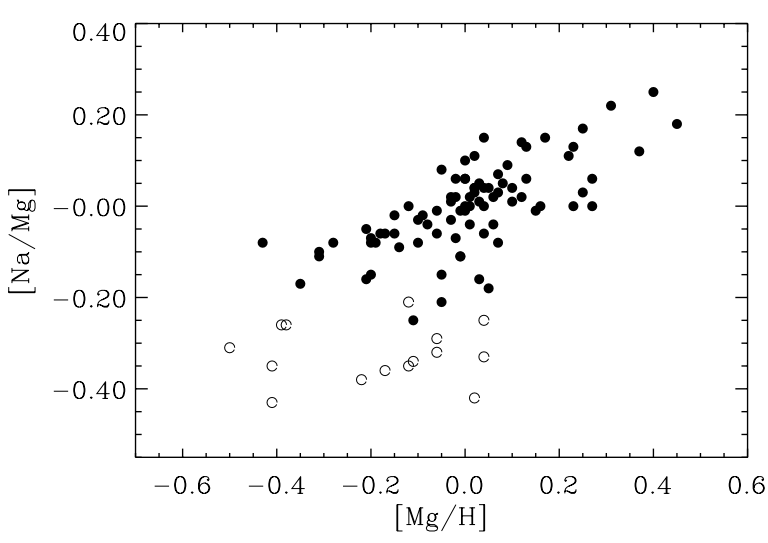

Fig. 4. $[\mathrm{Na} / \mathrm{Mg}]$ abundances ratios for NLTE analysis as a function of $[\mathrm{Mg} / \mathrm{H}]$. Symbols are the same as in Fig. 2.

sodium decreases with increasing metallicity in the range of $Z=0$ to $Z_{\odot}$. Also, $A G B$ stars are potential sites for the production of primary sodium (Mowlavi 1999). In this scenario synthesis of sodium can occur in hydrogen-burning shells during ON-cycling (Denissenkov \& Tout 2000). This process is supported by observations of red giant stars in globular clusters that appear to be sodium-rich (Kraft et al. 1997; Gratton et al. 2001).

Detailed modelling of the Galactic chemical evolution has been attempted by many authors (e.g., Timmes et al. 1995; Goswami \& Prantzos 2000). Based on Woosley \& Weaver's (1995) metallicity-dependent yields, the first authors calculate the behaviour of $[\mathrm{Na} / \mathrm{Fe}]$ as a function of metallicity. Their results predict that $[\mathrm{Na} / \mathrm{Fe}]$ decreases from $[\mathrm{Fe} / \mathrm{H}] \sim-3$ to $\sim-1.5$, while it increases from $[\mathrm{Fe} / \mathrm{H}] \sim-1.5$ to $\sim-0.5$ (see their Fig. 17). However using the same yields with an iron yield reduced by a factor of 2 , Goswami \& Prantzos' calculation explains the decline of $[\mathrm{Na} / \mathrm{Fe}]$ from $[\mathrm{Fe} / \mathrm{H}] \sim-1$ to $[\mathrm{Fe} / \mathrm{H}] \sim-2$ reasonably well. Their prediction suggests an ever-decreasing $[\mathrm{Na} / \mathrm{Fe}]$ toward the lower metallicity regime. They used a different initial mass function (IMF), and a different halo model.

Our observational results provide some implications for the nuclesynthesis of sodium. We note that in halo stars sodium is significantly underabundant relative to iron, and $[\mathrm{Na} / \mathrm{Fe}]$ (or $[\mathrm{Na} / \mathrm{Mg}]$ ) increases with metallicity (Fig. 5). In the region of overlapping metal abundances $[\mathrm{Na} / \mathrm{Fe}]$ shows a clear distinction between halo and thick-disk stars. The linear relationship indicates that sodium production is connected to the metallicity of the progenitor SN as suggested by Nissen \& Schuster (1997). This result agrees with nucleosynthesis calculations of massive stars by Umeda et al. (2000), but it is at variance with the calculations by Woosley \& Weaver (1995). We note that there are no extremely metal-poor stars in the sample of Gehren et al. (2004), whereas McWilliam et al. (1995) find that, for stars with $[\mathrm{Fe} / \mathrm{H}] \leq-3,[\mathrm{Na} / \mathrm{Fe}]$ rises with decreasing metal abundance. Based on this result Tsujimoto et al. (2002) argued that sodium yields decrease with increasing $Z$ for the very metal-poor stars. However, nearly all the stars are giants and the abundances were derived from the $\mathrm{Na} \mathrm{D}$ (5890/5896) lines, which are very sensitive to NLTE effects (see Sect. 5.1;
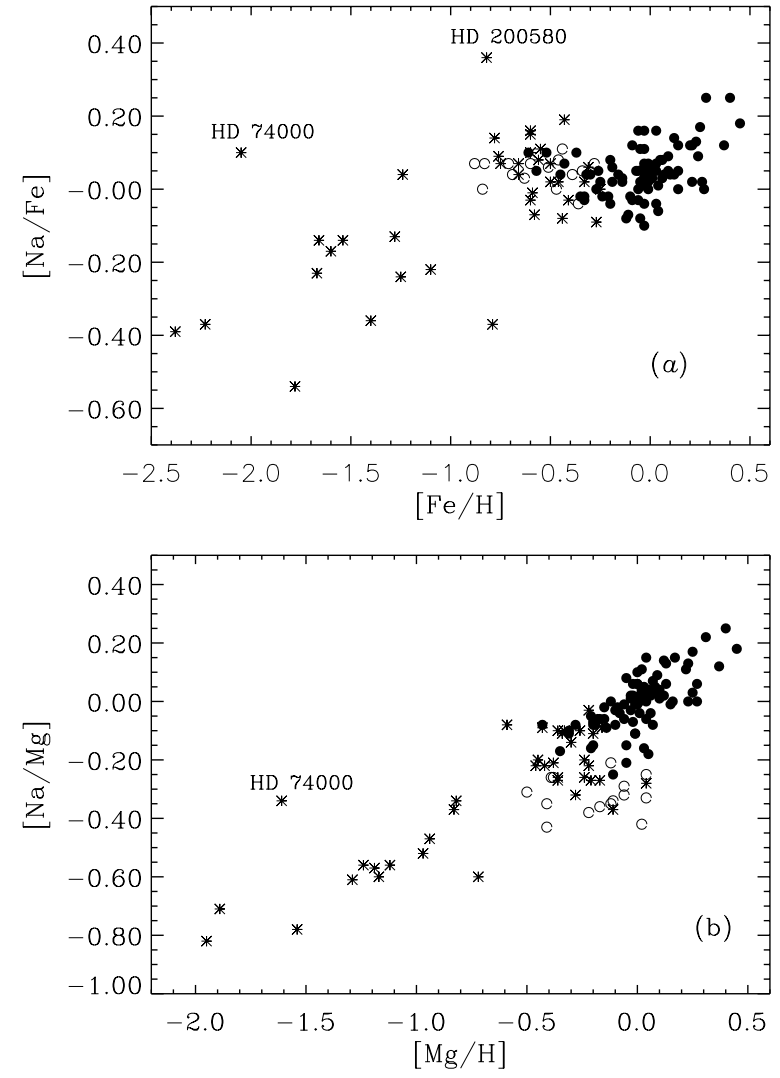

Fig. 5. Top: abundance ratios $[\mathrm{Na} / \mathrm{Fe}]$ for NLTE analysis as a function of $[\mathrm{Fe} / \mathrm{H}]$. Bottom: abundance ratios $[\mathrm{Na} / \mathrm{Mg}]$ for NLTE analysis as a function of $[\mathrm{Mg} / \mathrm{H}]$. Filled and open circles refer to the thin- and thickdisk stars in this paper, respectively. Asterisks refer to the stars from Gehren et al. (2004).

Takeda et al. 2003). There are two possibilities to explain the high $[\mathrm{Na} / \mathrm{Fe}]$ values of McWilliam et al.,

- NLTE corrections for Na D lines are large for metal-poor cool giants, so if it is considered, the $[\mathrm{Na} / \mathrm{Fe}]$ ratios would show an abundance deficiency (however, see Takeda et al. 2003);

- sodium is also produced in metal-poor giant stars (Goriely \& Siess 2001), which could explain why the Pb-stars also have a large sodium content (Van Eck et al. 2001).

A definitive confirmation of the latter assumption will require more detailed nucleosynthesis calculations. It is highly desirable to include the sample of ultra-low metallicity stars for establishing the behaviour of $[\mathrm{Na} / \mathrm{Fe}]$ in this region, which is important for investigating the history of the early Galaxy as well as for constructing the model of Galactic chemical evolution.

Based on [Eu/Ba] ratios, Mashonkina et al. (2003) suggested that during the active phase of the thick disk formation evolved low mass stars may enrich the interstellar gas. Our observational results show that $[\mathrm{Na} / \mathrm{Fe}]$ values are about solar, possibly being slightly overabundant in the thick-disk stars, which would confirm the suggestion that AGB stars may contribute some additional sodium. However, metallicitydependent SN yields of sodium can not be excluded.

While the origin of the "upturn" for the metal-rich stars is still unclear, Feltzing \& Gustafsson (1998) argued that 


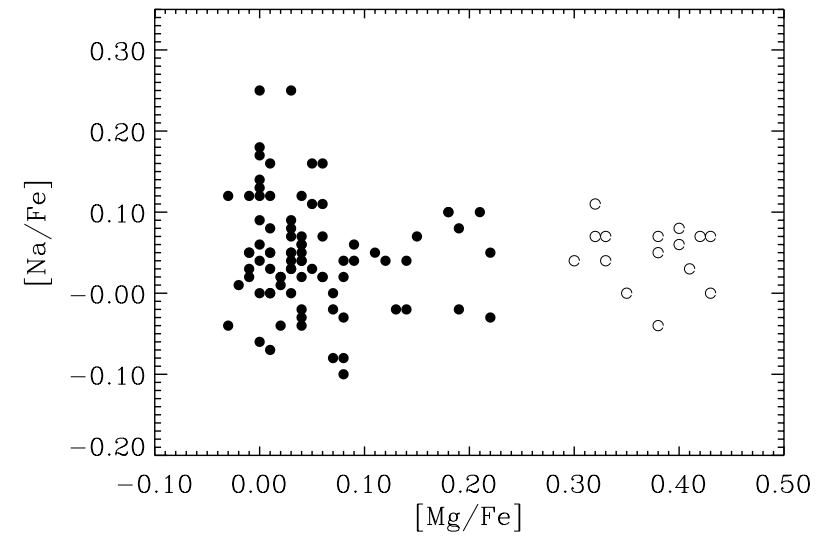

Fig. 6. $[\mathrm{Na} / \mathrm{Fe}]$ abundance ratios for NLTE analyses as a function of $[\mathrm{Mg} / \mathrm{Fe}]$. The meaning of symbols is the same as in Fig. 2.

metallicity-dependent SN yields may play a role. Though a rather insufficient fraction of stars supersolar metal abundances prevents us from making any definitive argument, we suggest that quite large amounts of Na may be produced by proton capture on ${ }^{22} \mathrm{Ne}$ nuclei for AGB stars (Denissenkov \& Denissenkova 1990).

\subsection{Abundance ratios, kinematics, ages and the Galactic stellar populations}

It is important to distinguish the individual membership of stars in our sample. We are aware that such a discussion requires more than the interpretation of abundance ratios alone. Following Fuhrmann's $(1998,2000)$ work we use $[\mathrm{Mg} / \mathrm{Fe}]=$ 0.25 as a first step to discriminate between thin- and thick-disk stars.

\subsubsection{Abundance ratios and population membership}

Fuhrmann (1998) attributed the scatter in $[\mathrm{Mg} / \mathrm{Fe}]$ to a mixing of thin- with thick-disk stars and a different chemical evolution of these two stellar populations (see also Gratton et al. 2000, 2003, for remarks on $\mathrm{O}$ and $\mathrm{Mg}$ with respect to $\mathrm{Fe}$ ). He suggested that the stars with higher $[\mathrm{Mg} / \mathrm{Fe}]$ at a given $[\mathrm{Fe} / \mathrm{H}]$ are old thick-disk stars (see also Reddy et al. 2003).

We show the distribution of $[\mathrm{Na} / \mathrm{Fe}]$ versus $[\mathrm{Mg} / \mathrm{Fe}]$ for the two populations of thick- and thin-disk stars in Fig. 6. Even comparing abundance alone, Fig. 6 suggests differences among the two populations: the thin-disk stars are well separated on the left side.

\subsubsection{Kinematic properties and population membership}

The original classification of stellar populations was based much more on kinematics than on anything else. It is therefore of high priority to check if kinematic properties and abundance ratios have more in common than a coarse change with the mean metal abundance itself. Fuhrmann (2000, 2002; see also Ryan \& Smith 2003) argues that in fact halo stars may constitute an extremely small minority of all Galactic stars, whereas most of the stars with intermediate kinematics instead belong to the thick-disk. Fuhrmann (2000) also suggested that there is some kinematical overlap of the disk populations. Therefore the determination of Galactic kinematics provides an important test.

Fuhrmann (2000) has discussed in more detail the kinematical aspects of our sample. The basic data are available in a number of catalogues which are electronically accessible (e.g. via http://www .ari.uni-heidelberg.de/aricns). The correction for the basic solar motion is that of Dehnen \& Binney (1998), namely, $U_{0} / V_{0} / W_{0}=10.00 / 5.25 / 7.17 \mathrm{~km} \mathrm{~s}^{-1}$. Whenever these data are not provided, we have calculated the space velocities following the model described by Chen et al. (2000).

The first inspection of Fig. 7 shows that the $V$ velocities of thin-disk stars tend to be significantly larger than those of the other populations, although there is some overlap between thin- and thick-disk stars. The two most exceptional cases are the thin-disk star HD $52711\left(V=-98 \mathrm{~km} \mathrm{~s}^{-1}\right)$ and the transition star HD $90508\left(V=-89 \mathrm{~km} \mathrm{~s}^{-1}\right)$, and we will come back to them later. The correlation between low $[\mathrm{Na} / \mathrm{Mg}]$ ratios and low $V$ or high peculiar space velocities $\left(v_{\text {spec }}\right)$ is also instructive (Fig. 8). The kinematic status in the Toomre diagram of our sample was shown by Fuhrmann (2000, in his Fig. 17).

We now come back to the transition stars HD 90508 $\left(U / V / W=31 /-89 / 30 \mathrm{~km} \mathrm{~s}^{-1}\right)$ and thin-disk object HD 52711 $\left(U / V / W=-17 /-98 /-13 \mathrm{~km} \mathrm{~s}^{-1}\right)$ : Fuhrmann (2000) argues that although HD 90508 shows thick-disk kinematics, its chemistry and age are more like thin-disk stars. HD 52711 is thought to be a thick-disk star based on its kinematics (e.g., Ibukiyama \& Arimoto 2002), however, it is very young (6.8 Gyr) and $[\mathrm{Mg} / \mathrm{Fe}]$ is about solar $(0.04)$, therefore it is also more like a thin-disk star.

\subsubsection{Stellar ages and population membership}

We now turn to the age aspects of analyzed stars. Gratton et al. (2000) concluded that there is a gap in the distribution of $[\mathrm{O} / \mathrm{Fe}]$ as expected from a sudden decrease in star formation during the transition from the thick- to thin-disk phase. Fuhrmann (2000) suggested that the key feature to identify thick-and thin-disk stars individually is provided by the stellar ages, while there is a considerable age overlap between halo and thick-disk stars.

Using the stellar effective temperatures together with the absolute magnitudes based on Hipparcos parallaxes, masses and approximate ages can be interpolated according to $[\mathrm{Fe} / \mathrm{H}]$ and $[\alpha / \mathrm{Fe}]$ from adequate tracks of stellar evolution. Such calculations have recently become available through the work of VandenBerg et al. (2000, 2002) and Yi et al. (2003). In this paper we adopt the Yonsei-Yale isochrones (Yi et al. 2003) since the evolutionary tracks of VandenBerg contain only metal-poor $([\mathrm{Fe} / \mathrm{H}] \leq-0.31)$ and low mass stars $\left(M_{\text {sun }} \leq 1.20\right)$. The Yonsei-Yale isochrones are calculated with new OPAL opacities and Kurucz model atmospheres for a set of metallicities $Z=0.00001,0.0001,0.0004,0.001,0.004,0.007,0.01,0.02$, $0.04,0.06,0.08$, and $[\alpha / \mathrm{Fe}]=0.0,0.3,0.6$. The mixing length and helium enrichment rate were fixed to $\alpha \equiv l / H_{\mathrm{p}}=1.7431$ 

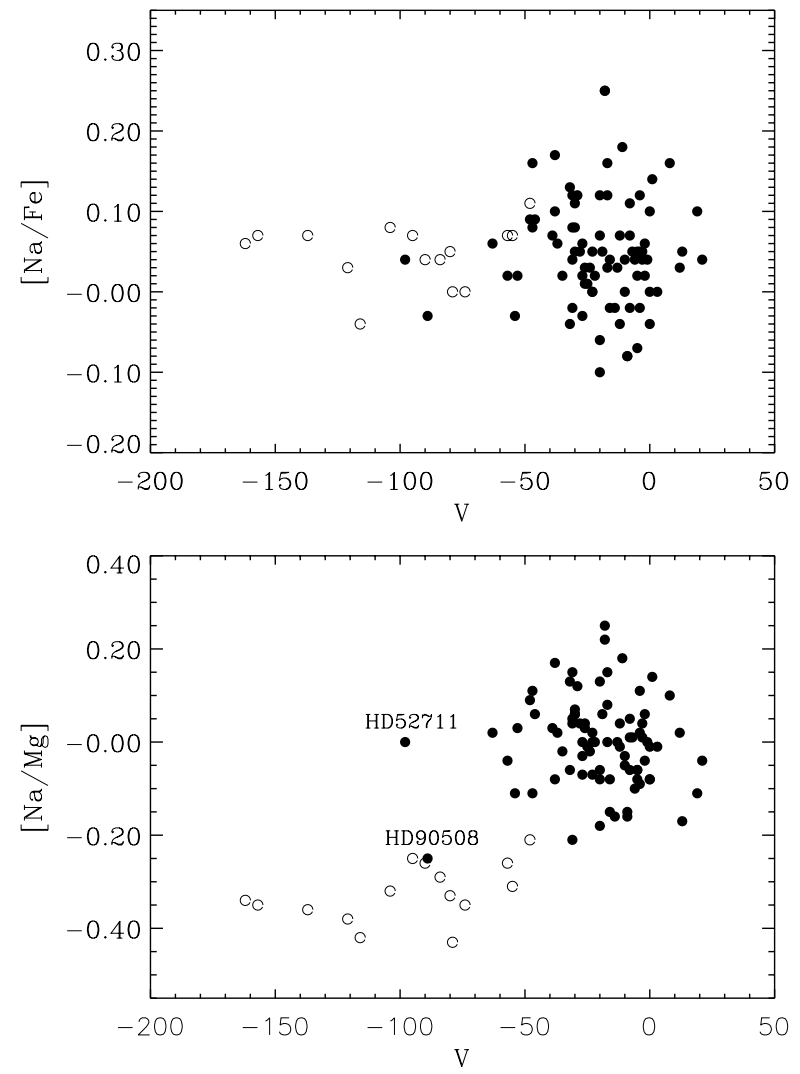

Fig. 7. Correlation between $\mathrm{Na}$ abundance ratios and orbital $V$ velocity components. Symbols are as in Fig. 2.

and $\Delta \mathrm{Y} / \Delta Z=2.0$, respectively. The full set of stellar models and a FORTRAN package that works for mass, metallicity and $\alpha$-enhancement interpolation are available from the authors.

The ages derived here are based on the assumption that $[\mathrm{O} / \mathrm{Mg}]=0$. From Fig. 9 we can see that all the thindisk stars do not exceed $\sim 9$ Gyr, whereas with the exclusion of HD 165401 all thick-disk stars are older than $\sim 9$ Gyr. Fuhrmann (1998) identified this star as a thick-disk member with an age of about $10 \mathrm{Gyr}$, but in his Paper II (Fuhrmann 2000) he noted that it is a chromospherically active, highvelocity star, so it was excluded from his later analysis.

As already pointed out by VandenBerg et al. (2002) and iterated by Gehren et al. (2004), a significantly higher $[\mathrm{Mg} / \mathrm{Fe}]$ ratio could reduce the ages of thick-disk and halo stars by as much as 3 Gyr. For comparison, we also calculated the ages for these stars without $\alpha$-enhancement. Then our result is fairly in agreement with Fuhrmann's, namely that all the thick-disk (excluding HD 165401) and halo stars are older than 12 Gyr, while all the thin-disk stars are younger than 9.5 Gyr except the three transition stars (HD 90508, HD 143761 and HD 187923). Therefore Fuhrmann's result that the key feature to identify thick- and thin-disk stars individually is provided by the stellar ages still holds. However, the 3 Gyr long star formation gap between thin- and thick-disk is not confirmed here.

The three transition stars (HD 90508, HD 143761 and HD 187923) have ages about $8.8 \mathrm{Gyr}$, thus they are as old as the oldest thin-disk stars. They would be about 10.5 Gyr old if no $\alpha$-enhancement were considered.
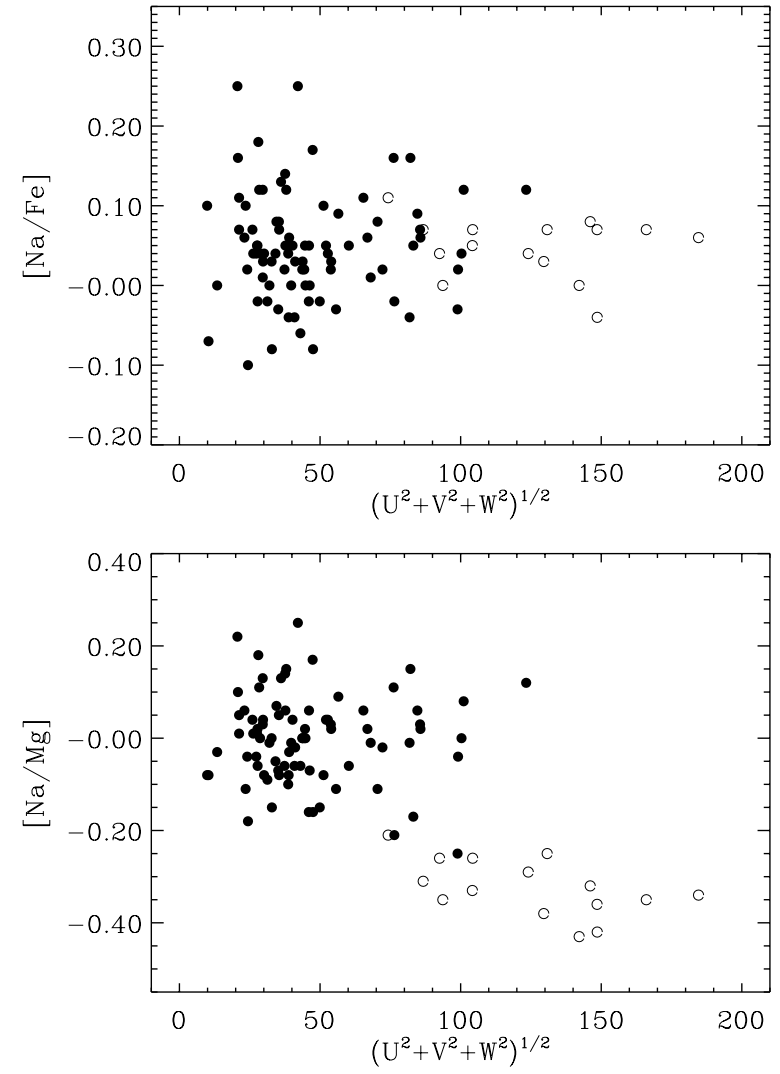

Fig. 8. Correlation between $[\mathrm{Na} / \mathrm{Fe}]$ abundance ratios and peculiar space velocities $v_{\text {spec }}=\left(U^{2}+V^{2}+W^{2}\right)^{1 / 2}($ top $)$, and correlation between $[\mathrm{Na} / \mathrm{Mg}]$ abundance ratios and peculiar space velocities $v_{\text {spec }}=$ $\left(U^{2}+V^{2}+W^{2}\right)^{1 / 2}$ (bottom). The meaning of the symbols is the same as in Fig. 2.

We also note that the age of the thick-disk star HD 6582 seems to be very high (>15 Gyr); Gehren et al. (2004) have discussed this in detail.

\section{Conclusions}

We have determined sodium abundances for some 90 nearby stars, spanning the range $-0.9<[\mathrm{Fe} / \mathrm{H}]<+0.4$. All abundances are derived from NLTE statistical equilibrium calculations. Based on our results we come to the following conclusions:

1. The $[\mathrm{Na} / \mathrm{Fe}]$ ratios are about solar for thin- and thick-disk stars. The "upturn" of $[\mathrm{Na} / \mathrm{Fe}]$ for metal-rich stars is reproduced.

2. The NLTE effects are different from line to line. The $6154 / 6160$ lines are comparatively less sensitive to NLTE effects, while the Na D and near-IR line pairs show large NLTE effects. Large departures from LTE appear for warm metal-poor subgiant stars.

3. Our results suggest that the sodium yields increase with metallicity, and quite large amounts of sodium may be produced by AGB stars.

4. Combined with the abundance ratios, kinematics and stellar ages, we identify individual thick- and thin-disk stars. 

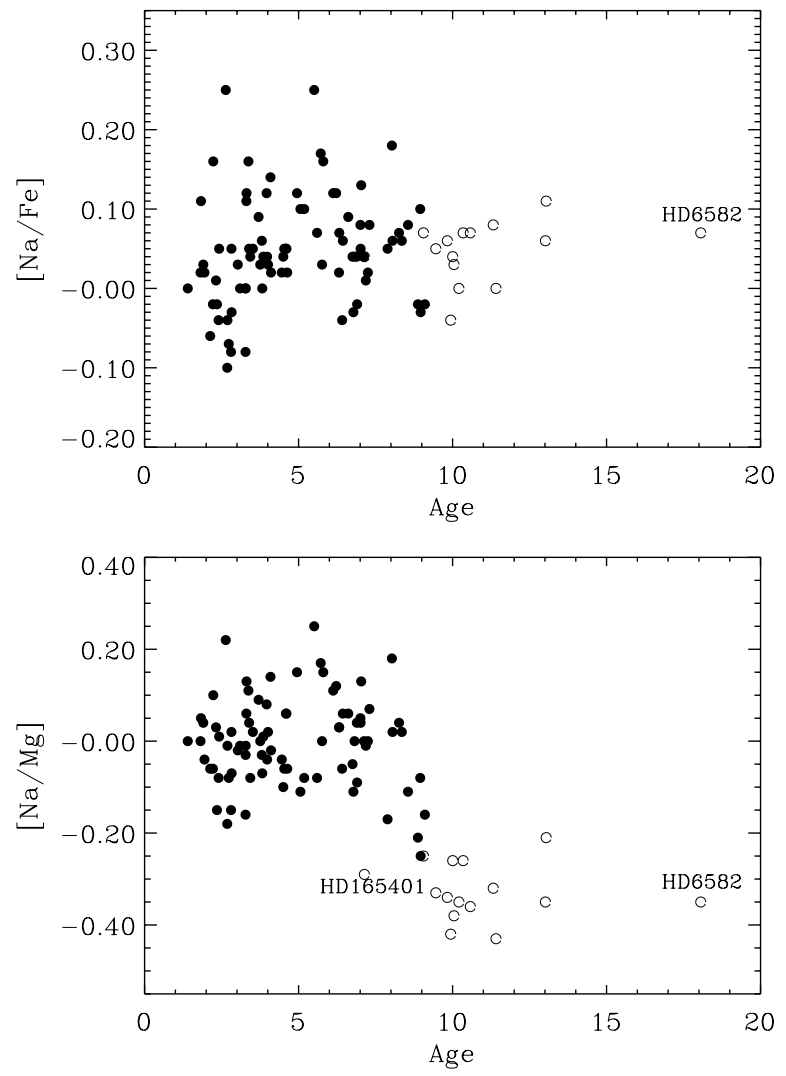

Fig. 9. $[\mathrm{Na} / \mathrm{Fe}]$ ratios vs. stellar ages (top), and $[\mathrm{Na} / \mathrm{Mg}]$ abundance ratios vs. stellar ages (bottom). Ages are in Gyr. Symbols have the same meaning as in Fig. 2.

Fuhrmann's suggestion that stellar ages provide a key feature in discriminating the two disk populations is supported.

It would be important to perform NLTE abundance determinations for both $\mathrm{Mg}$ and $\mathrm{Al}$ for these program stars to confirm our identification, because both $\mathrm{Al}$ and $\mathrm{Mg}$ show large NLTE effects for metal-poor stars (Gehren et al. 2004). It will be also necessary to investigate some extremely metal-poor stars.

Acknowledgements. S.J.R. thanks the Institute of Astronomy and Astrophysics of Munich University for warm hospitality during a productive stay in 2002. The authors thank Klaus Fuhrmann for his kind permission to use the reduced FOCES spectra of the stars investigated in this paper. This research was supported by the Deutsche Forschungsgemeinschaft under contract Ge 490/26-1 and by the National Natural Science Foundation of China under grants No.10173014, No.10173028 and NKBRSF 1999075406.

\section{References}

Anstee, S. D., \& O'Mara, B. J. 1991, MNRAS, 253, 549 Anstee, S. D., \& O'Mara, B. J. 1995, MNRAS, 276, 859 Arnett, W. D. 1971, ApJ, 166, 153

Baumüller, D., Butler, K., \& Gehren, T. 1998, A\&A, 338, 637

Baumüller, D., \& Gehren, T. 1997, A\&A, 325, 1088

Butler, K. 1993, unpublished

Butler, K., \& Giddings, J. 1985, Newsletter on the analysis of astronomical spectra No. 9, University of London

Butler, K., Mendoza, C., \& Zeippen, C. J. 1993, J. Phys. B, 26, 4409

Carretta, E., Gratton, R. G., \& Sneden, C. 2000, A\&A, 356, 238
Chen, Y. Q., Nissen, P. E., Zhao, G., et al. 2000, A\&AS, 141, 491

Dehnen, W., \& Binney, J. J. 1998, MNRAS, 298, 387

Denissenkov, P. A., \& Denissenkova, S. N. 1990, SvA Lett., 16, 275

Denissenkov, P. A., \& Tout, C. A. 2000, MNRAS, 316, 395

Edvardsson, B., Andersen, J., Gustafsson, B., et al. 1993, A\&A, 275, 101

Feltzing, S., \& Gustafsson, B. 1998, A\&AS, 129, 237

Fuhrmann, K. 1998, A\&A, 338, 161

Fuhrmann, K. 2000, http://www . xray .mpe.mpg.de/ fuhrmann

Fuhrmann, K., Axer, M., \& Gehren, T. 1993, A\&A, 271, 451

Fuhrmann, K. 2002, New Astron., 7, 161

Fuhrmann, K. 2004, AN, 325, 3

Fuhrmann, K., Pfeiffer, M., Frank, C., et al. 1997, A\&A, 323, 909

Fulbright, J. P. 2000, AJ, 120, 1841

Fulbright, J. P. 2002, AJ, 123, 404

Gehren, T., Butler, K., Mashonkina, L., et al. 2001, A\&A, 366, 981

Gehren, T., Liang, Y. C., Shi, J. R., et al. 2004, A\&A, 413, 1045

Gilmore, G. 1995, in Stellar Populations ed. P. C. van der Kruit, \& G. Gilmore (Dordrecht: Kluwer), IAU Symp., 164, 99

Goriely, S., \& Siess, L. 2001, A\&A, 378, L25

Goswami, A., \& Prantzos, N. 2000, A\&A, 359, 191

Gray, D. F. 1984, ApJ, 281, 719

Gratton, R. G., Bonifacio, P., Bragaglia, A., et al. 2001, A\&A, 369, 87

Gratton, R. G., Carretta, E., \& Castelli, F. 1996, A\&A, 314, 191

Gratton, R. G., Carretta, E., Claudi, R., et al. 2003, A\&A, 404, 187

Gratton, R. G., Carretta, E., Matteucci, F., et al. 2000, A\&A, 358, 671

Ibukiyama, A., \& Arimoto, N. 2002, A\&A, 394, 927

Korn, A., Shi, J. R., \& Gehren, T. 2003, A\&A, 407, 691

Kraft, R. P., Sneden, C., Smith, G. H., et al. 1997, AJ, 113, 279

Kurucz, R. L., Furenlid, I., Brault, J., et al. 1984, Solar Flux Atlas from 296 to $1300 \mathrm{~nm}$, Nat. Solar Obs., Sunspot, New Mexico

Limongi, M., Straniero, O., \& Chieffi, A. 1998, in Stellar Evolution, Stellar Explosions and Galactic Evolution, ed. A. Mezzacappa, London, Inst. Phys., 385

Martin, W. C., \& Zalubas, R. 1981, J. Phys. Chem. Rev. Data, 153

Mashonkina, L., Gehren, T., Travaglio, C., et al. 2003, A\&A, 397, 275

McWilliam, A., Preston, G. W., Sneden, C., et al. 1995, AJ, 109, 2736

Mowlavi, N. 1999, A\&A, 350, 73

Nissen, P. E., \& Schuster, W. J. 1997, A\&A, 326, 751

Nomoto, K., Iwamoto, K., Nakasato, N., et al. 1997, Nucl. Phys. A, $621,467 \mathrm{c}$

Pfeiffer, M. J., Frank, C., Baumüller, D., et al. 1998, A\&AS, 130, 381

Pilachowski, C. A., Sneden, C., \& Kraft, R. P. 1996, AJ, 111, 1689

Prochaska, J. X., Naumov, S. O., Carney, B. W., et al. 2000, AJ, 120, 2513

Reddy, B. E., Tomkin, J., Lambert, D. L., et al. 2003, MNRAS, 340, 304

Ryan, S. G., \& Smith, I. M. 2003, MNRAS, 341, 199

Stephens, A. 1999, AJ, 117, 1771

Takeda, Y., Zhao, G., Takeda-Hidai, M., et al. 2003, CJA\&A, 3, 316

Timmes, F. X., Woosley, S. E., \& Weaver, T. A. 1995, ApJS, 98, 617

Tsujimoto, T., Nomoto, K., Yoshii, Y., et al. 1995, MNRAS, 277, 945

Tsujimoto, T., Shigayama, T., \& Yoshii, Y. 2002, ApJ, 565, 1011

Umeda, H., Nomoto, K., \& Nakamura, T. 2000, in The first Stars, ed. A. Weiss, T. Abel, \& V. Hill (Heidelberg: Springer), 150

VandenBerg, D. A., Swenson, F. J., Rogers, F. J., et al. 2000, ApJ, 532, 430

VandenBerg, D. A., Richard, O., Michaud, G., et al. 2002, ApJ, 571, 487

Van Eck, S., Goriely, S., Jorisse, A., et al. 2001, Nature, 412, 793

Woosley, S. E., \& Weaver, T. A. 1995, ApJS, 101, 181

Yi, S. K., Kim, Y. C., \& Demarque, P. 2003, ApJS, 144, 259

Zhao, G., \& Gehren, T. 2000, A\&A, 362, 1077 


\section{Online Material}


J. R. Shi et al.: Sodium abundances in nearby disk stars, Online Material p 2

Table 2. Atmospheric parameters and sodium abundances of the program stars. Rotational velocities and macroturbulence data are the same as in Fuhrmann $(1998,2000)$.

\begin{tabular}{|c|c|c|c|c|c|c|}
\hline HD & $T_{\text {eff }}$ & $\log g$ & {$[\mathrm{Fe} / \mathrm{H}]$} & $\xi$ & $\begin{array}{r}{[\mathrm{Na} / \mathrm{Fe}]} \\
\mathrm{LTE}\end{array}$ & $\begin{array}{r}{[\mathrm{Na} / \mathrm{Fe}]} \\
\mathrm{NLTE}\end{array}$ \\
\hline 400 & 6149 & 4.06 & -0.25 & 1.31 & 0.11 & 0.03 \\
\hline 3079 & 6050 & 4.17 & -0.14 & 1.42 & 0.09 & 0.02 \\
\hline 4614 & 5939 & 4.33 & -0.30 & 1.06 & 0.09 & 0.04 \\
\hline 5015 & 6045 & 3.90 & -0.02 & 1.39 & 0.12 & 0.04 \\
\hline 6582 & 5387 & 4.45 & -0.83 & 0.89 & 0.14 & 0.07 \\
\hline 6920 & 5838 & 3.48 & -0.05 & 1.35 & 0.08 & 0.02 \\
\hline 9407 & 5663 & 4.42 & 0.03 & 0.87 & 0.09 & 0.05 \\
\hline 9826 & 6107 & 4.01 & 0.09 & 1.40 & 0.13 & 0.04 \\
\hline 10697 & 5614 & 3.96 & 0.10 & 1.04 & 0.11 & 0.04 \\
\hline 16141 & 5737 & 3.92 & 0.02 & 1.24 & 0.14 & 0.06 \\
\hline 16895 & 6248 & 4.20 & -0.01 & 1.42 & 0.08 & 0.00 \\
\hline 18757 & 5714 & 4.34 & -0.28 & 0.95 & 0.13 & 0.06 \\
\hline 18803 & 5657 & 4.39 & 0.14 & 0.87 & 0.12 & 0.05 \\
\hline 19373 & 5966 & 4.15 & 0.03 & 1.23 & 0.24 & 0.16 \\
\hline 19994 & 6087 & 3.97 & 0.14 & 1.19 & 0.20 & 0.12 \\
\hline 20619 & 5706 & 4.48 & -0.20 & 0.90 & 0.02 & -0.04 \\
\hline 22879 & 5867 & 4.26 & -0.84 & 1.21 & 0.06 & 0.00 \\
\hline 25457 & 6246 & 4.32 & 0.06 & 1.25 & 0.10 & 0.03 \\
\hline 30649 & 5816 & 4.28 & -0.47 & 1.18 & 0.06 & 0.00 \\
\hline 30743 & 6298 & 4.03 & -0.45 & 1.64 & 0.15 & 0.04 \\
\hline 34411 & 5875 & 4.18 & 0.03 & 1.08 & 0.13 & 0.07 \\
\hline 37124 & 5610 & 4.44 & -0.44 & 0.89 & 0.17 & 0.11 \\
\hline 43042 & 6444 & 4.23 & 0.04 & 1.52 & 0.01 & -0.06 \\
\hline 43386 & 6480 & 4.27 & -0.06 & 1.56 & 0.26 & 0.16 \\
\hline 52711 & 5887 & 4.31 & -0.16 & 1.04 & 0.10 & 0.04 \\
\hline 58855 & 6309 & 4.16 & -0.32 & 1.38 & 0.14 & 0.04 \\
\hline 61421 & 6600 & 3.96 & -0.03 & 1.83 & 0.19 & 0.11 \\
\hline 62301 & 5938 & 4.06 & -0.69 & 1.28 & 0.11 & 0.04 \\
\hline 67228 & 5847 & 3.93 & 0.12 & 1.18 & 0.22 & 0.14 \\
\hline 69611 & 5821 & 4.18 & -0.60 & 1.22 & 0.14 & 0.07 \\
\hline 75732 & 5336 & 4.47 & 0.40 & 0.76 & 0.28 & 0.25 \\
\hline 90508 & 5802 & 4.35 & -0.33 & 1.02 & 0.03 & -0.02 \\
\hline 95128 & 5892 & 4.27 & 0.00 & 1.01 & 0.12 & 0.06 \\
\hline 102158 & 5758 & 4.24 & -0.46 & 1.13 & 0.14 & 0.08 \\
\hline 102870 & 6085 & 4.04 & 0.14 & 1.38 & 0.08 & 0.00 \\
\hline 109358 & 5863 & 4.36 & -0.21 & 1.12 & 0.03 & -0.02 \\
\hline 114710 & 6006 & 4.30 & -0.03 & 1.12 & 0.08 & 0.03 \\
\hline 114762 & 5934 & 4.11 & -0.71 & 1.14 & 0.13 & 0.07 \\
\hline 117176 & 5483 & 3.82 & -0.09 & 1.02 & 0.04 & -0.03 \\
\hline 120136 & 6360 & 4.17 & 0.27 & 1.56 & 0.07 & 0.00 \\
\hline 121370 & 6023 & 3.76 & 0.28 & 1.40 & 0.35 & 0.25 \\
\hline 121560 & 6144 & 4.27 & -0.43 & 1.26 & 0.14 & 0.07 \\
\hline 122742 & 5537 & 4.43 & -0.01 & 0.78 & 0.12 & 0.07 \\
\hline 124850 & 6112 & 3.78 & -0.06 & 1.48 & 0.10 & -0.03 \\
\hline 126053 & 5691 & 4.45 & -0.35 & 0.96 & 0.03 & -0.02 \\
\hline 127334 & 5691 & 4.22 & 0.21 & 0.97 & 0.18 & 0.12 \\
\hline 130322 & 5394 & 4.55 & 0.04 & 0.79 & 0.05 & 0.01 \\
\hline 130819 & 6598 & 4.18 & -0.10 & 1.76 & 0.06 & -0.02 \\
\hline 132254 & 6220 & 4.15 & -0.03 & 1.46 & 0.10 & 0.03 \\
\hline
\end{tabular}

Table 2. continued.

\begin{tabular}{|c|c|c|c|c|c|c|}
\hline HD & $T_{\text {eff }}$ & $\log g$ & {$[\mathrm{Fe} / \mathrm{H}]$} & $\xi$ & $\begin{array}{r}{[\mathrm{Na} / \mathrm{Fe}]} \\
\mathrm{LTE}\end{array}$ & $\begin{array}{r}{[\mathrm{Na} / \mathrm{Fe}]} \\
\mathrm{NLTE}\end{array}$ \\
\hline 134987 & 5740 & 4.25 & 0.25 & 1.02 & 0.24 & 0.17 \\
\hline 136064 & 6116 & 3.86 & -0.05 & 1.41 & 0.18 & 0.11 \\
\hline 136202 & 6104 & 3.80 & -0.09 & 1.45 & 0.21 & 0.12 \\
\hline 137052 & 6283 & 3.94 & -0.03 & 1.89 & -0.02 & -0.09 \\
\hline 141004 & 5864 & 4.09 & -0.03 & 1.05 & 0.08 & 0.01 \\
\hline 142373 & 5841 & 3.84 & -0.57 & 1.24 & 0.13 & 0.05 \\
\hline 142860 & 6254 & 4.02 & -0.19 & 1.36 & 0.10 & 0.02 \\
\hline 143761 & 5821 & 4.12 & -0.24 & 1.10 & 0.05 & -0.02 \\
\hline 145675 & 5334 & 4.51 & 0.45 & 0.81 & 0.23 & 0.18 \\
\hline 146233 & 5786 & 4.36 & 0.01 & 0.98 & 0.09 & 0.03 \\
\hline 150680 & 5814 & 3.72 & -0.03 & 1.38 & 0.25 & 0.16 \\
\hline 153597 & 6275 & 4.36 & -0.11 & 1.48 & -0.01 & -0.07 \\
\hline 154345 & 5507 & 4.57 & -0.03 & 0.70 & 0.00 & -0.04 \\
\hline 157214 & 5735 & 4.24 & -0.34 & 1.00 & 0.12 & 0.06 \\
\hline 157347 & 5643 & 4.31 & -0.01 & 0.90 & 0.08 & 0.02 \\
\hline 159222 & 5845 & 4.28 & 0.09 & 1.05 & 0.15 & 0.09 \\
\hline 161797 & 5596 & 3.93 & 0.23 & 1.17 & 0.23 & 0.16 \\
\hline 162003 & 6423 & 4.06 & -0.07 & 1.67 & 0.17 & 0.05 \\
\hline 162004 & 6203 & 4.24 & -0.06 & 1.29 & 0.11 & 0.04 \\
\hline 163989 & 6116 & 3.62 & -0.14 & 1.61 & 0.13 & 0.03 \\
\hline 165401 & 5811 & 4.41 & -0.39 & 1.10 & 0.10 & 0.04 \\
\hline 165908 & 5994 & 4.01 & -0.61 & 1.25 & 0.17 & 0.09 \\
\hline 168009 & 5785 & 4.23 & -0.03 & 1.03 & 0.13 & 0.06 \\
\hline 168151 & 6357 & 4.02 & -0.33 & 1.68 & 0.05 & -0.02 \\
\hline 173667 & 6305 & 3.99 & 0.03 & 1.60 & 0.09 & -0.04 \\
\hline 176377 & 5860 & 4.43 & -0.27 & 0.91 & 0.04 & 0.00 \\
\hline 178428 & 5673 & 4.23 & 0.12 & 0.96 & 0.10 & 0.04 \\
\hline 182572 & 5610 & 4.19 & 0.37 & 1.01 & 0.19 & 0.12 \\
\hline 184499 & 5828 & 4.13 & -0.51 & 1.17 & 0.14 & 0.05 \\
\hline 186408 & 5805 & 4.26 & 0.06 & 1.03 & 0.14 & 0.08 \\
\hline 186427 & 5766 & 4.29 & 0.05 & 0.89 & 0.14 & 0.08 \\
\hline 187013 & 6312 & 4.09 & -0.05 & 1.46 & 0.02 & -0.08 \\
\hline 187691 & 6088 & 4.07 & 0.07 & 1.35 & 0.13 & 0.04 \\
\hline 187923 & 5713 & 3.97 & -0.20 & 1.19 & 0.16 & 0.08 \\
\hline 190360 & 5588 & 4.27 & 0.24 & 0.98 & 0.15 & 0.09 \\
\hline 190406 & 5937 & 4.35 & -0.01 & 1.10 & 0.10 & 0.05 \\
\hline 204155 & 5829 & 4.12 & -0.63 & 1.18 & 0.12 & 0.03 \\
\hline 207978 & 6313 & 3.94 & -0.52 & 1.57 & 0.20 & 0.10 \\
\hline 209458 & 6082 & 4.33 & -0.06 & 1.15 & 0.05 & 0.00 \\
\hline 210277 & 5541 & 4.42 & 0.26 & 0.73 & 0.07 & 0.02 \\
\hline 210855 & 6176 & 3.81 & 0.21 & 1.61 & 0.11 & 0.02 \\
\hline 215648 & 6110 & 3.85 & -0.37 & 1.69 & 0.21 & 0.10 \\
\hline 216385 & 6212 & 3.85 & -0.26 & 1.54 & 0.15 & 0.05 \\
\hline 217014 & 5793 & 4.33 & 0.20 & 0.95 & 0.19 & 0.12 \\
\hline 218470 & 6407 & 4.07 & -0.12 & 1.85 & 0.05 & -0.08 \\
\hline 219623 & 6140 & 4.23 & 0.01 & 1.30 & 0.11 & 0.05 \\
\hline 221830 & 5749 & 4.19 & -0.36 & 1.14 & 0.02 & -0.04 \\
\hline 222368 & 6157 & 3.95 & -0.19 & 1.51 & 0.15 & 0.06 \\
\hline G78-1 & 5895 & 4.11 & -0.88 & 1.14 & 0.17 & 0.07 \\
\hline
\end{tabular}


J. R. Shi et al.: Sodium abundances in nearby disk stars, Online Material p 3

Table 3. Abundance differences between NLTE and LTE obtained by fitting calculated LTE equivalent widths of Na I lines using the same parameters for various stellar model atmospheres based on the line-blanketed model grid. Results refer to $\Delta[\mathrm{Na} / \mathrm{Fe}]=[\mathrm{Na} / \mathrm{Fe}]_{\mathrm{NLTE}}-[\mathrm{Na} / \mathrm{Fe}]_{\mathrm{LTE}}$. No entries are given for extremely weak lines. The calculations all refer to $\xi=1 \mathrm{~km} \mathrm{~s}^{-1}$ and $[\mathrm{Na} / \mathrm{Fe}]=0$.

\begin{tabular}{rrrrrrrrrrrr}
\hline \hline$T_{\text {eff }}$ & $\log g$ & {$[\mathrm{Fe} / \mathrm{H}]$} & 5682 & 5688 & 5890 & 5896 & 6154 & 6160 & 8183 & 8194 & $\overline{\Delta[\mathrm{Na} / \mathrm{Fe}]}$ \\
\hline 5200 & 4.50 & 0.0 & -0.08 & -0.07 & -0.04 & -0.04 & -0.04 & -0.05 & -0.15 & -0.15 & $-0.08 \pm 0.05$ \\
5200 & 4.50 & -1.0 & -0.06 & -0.06 & -0.07 & -0.08 & -0.04 & -0.04 & -0.24 & -0.24 & $-0.10 \pm 0.07$ \\
5200 & 4.50 & -2.0 & & & -0.14 & -0.17 & & & & & $-0.15 \pm 0.02$ \\
5200 & 4.50 & -3.0 & & & -0.17 & -0.17 & & & & & $-0.17 \pm 0.00$ \\
5200 & 3.50 & 0.0 & -0.14 & -0.14 & -0.04 & -0.06 & -0.06 & -0.06 & -0.31 & -0.31 & $-0.14 \pm 0.09$ \\
5200 & 3.50 & -1.0 & & & -0.14 & -0.16 & & & -0.42 & -0.46 & $-0.29 \pm 0.15$ \\
5200 & 3.50 & -2.0 & & & -0.31 & -0.34 & & & & & $-0.32 \pm 0.02$ \\
5200 & 3.50 & -3.0 & & & -0.36 & -0.28 & & & & & $-0.32 \pm 0.04$ \\
5780 & 4.50 & 0.0 & -0.08 & -0.08 & -0.05 & -0.06 & -0.04 & -0.05 & -0.20 & -0.21 & $-0.10 \pm 0.05$ \\
5780 & 4.50 & -1.0 & -0.06 & -0.07 & -0.16 & -0.18 & -0.05 & -0.05 & -0.27 & -0.29 & $-0.14 \pm 0.08$ \\
5780 & 4.50 & -2.0 & -0.05 & -0.05 & -0.34 & -0.32 & -0.05 & -0.05 & -0.10 & -0.12 & $-0.13 \pm 0.10$ \\
5780 & 4.50 & -3.0 & & & -0.23 & -0.16 & & & & & $-0.19 \pm 0.04$ \\
5780 & 3.50 & 0.0 & -0.17 & -0.18 & -0.10 & -0.10 & -0.06 & -0.08 & -0.43 & -0.43 & $-0.19 \pm 0.12$ \\
5780 & 3.50 & -1.0 & -0.11 & -0.11 & -0.33 & -0.35 & & & -0.39 & -0.43 & $-0.29 \pm 0.12$ \\
5780 & 3.50 & -2.0 & & & -0.56 & -0.52 & & & & & $-0.54 \pm 0.02$ \\
5780 & 3.50 & -3.0 & & & -0.27 & -0.17 & & & & & $-0.22 \pm 0.05$ \\
6500 & 4.50 & 0.0 & -0.07 & -0.09 & -0.11 & -0.15 & -0.03 & -0.04 & -0.28 & -0.30 & $-0.13 \pm 0.08$ \\
6500 & 4.50 & -1.0 & & & -0.38 & -0.41 & & & -0.25 & -0.30 & $-0.33 \pm 0.06$ \\
6500 & 4.50 & -2.0 & & & -0.45 & -0.38 & & & & & $-0.41 \pm 0.04$ \\
6500 & 4.50 & -3.0 & & & -0.13 & -0.10 & & & & & $-0.11 \pm 0.02$ \\
6500 & 3.50 & 0.0 & -0.12 & -0.13 & -0.22 & -0.31 & -0.05 & -0.06 & -0.47 & -0.52 & $-0.23 \pm 0.15$ \\
6500 & 3.50 & -1.0 & & & -0.64 & -0.61 & & & -0.32 & -0.40 & $-0.49 \pm 0.02$ \\
6500 & 3.50 & -2.0 & & & -0.54 & -0.43 & & & & & $-0.49 \pm 0.06$ \\
6500 & 3.50 & -3.0 & & & -0.13 & -0.09 & & & & & $-0.11 \pm 0.02$ \\
\hline
\end{tabular}

